\title{
Baseband Design of Broadband Wireless ad hoc Network and Cross-layer Optimization of Transmission Power Control
}

\author{
WeiChun $^{1}$, LiMing ${ }^{2, a}$ \\ ${ }^{1}$ School of Electronic and Information Engineering, Wuhan Donghu University, Wuhan 430211, \\ China; \\ ${ }^{2}$ Information management center, Air Force Early Warning Academy, Wuhan 430019, China \\ aAFEWA_LM@163.com
}

Keywords: wireless ad hoc, transmission power, load capacity, cross layer optimization, throughput efficiency

\begin{abstract}
The capacity and transmission power of the wireless ad hoc networks are limited, so the key to study in the field of ad hoc networks is how to use the capacity of the nodes and improve the performance of the network. Based on the research of traditional layered protocol, a new cross layer optimization method is proposed, which is based on the network capacity and transmission power. The OFDM technology is introduced into the self-organization network. The MAC protocol can be transmitted to the transmission layer, so that the TCP protocol can distinguish the network congestion. Using the OPNET software, the simulation experiments are carried out. The results show that the transmission power of the cross layer optimization method can effectively optimize the network, reduce the time to wait for the time, improve the average throughput efficiency, and achieve the same effect as the packet loss.
\end{abstract}

\section{Introduction}

Wireless ad hoc network is a multi hop temporary autonomous system, which is composed of a group of mobile nodes with wireless transceiver. It is not required to set up the network infrastructure. The network is flexible and can be multi hop topology. However, the capacity of the network is limited and the transmission power is limited, so it is needed to improve the performance of ad hoc networks by using network optimization. How to save the energy cost of nodes, improve the capacity of link load, and efficient utilization of the network nodes, are the key problems which restrict the research and development of self organization network.

\section{Design principles of capacity and power control for Wireless Ad Hoc Networks}

Wireless ad hoc network protocol stack can be divided into 5 layers, including physical hardware layer, data link layer, network layer, transport layer and application layer, network capacity and transmission power control is the premise of ensuring communication quality, by increasing the network load, adjust the transmission power, realize the network power consumption, improve the network throughput efficiency, based on transmission power cross layer optimization of network control framework structure is shown in Figure 1. 


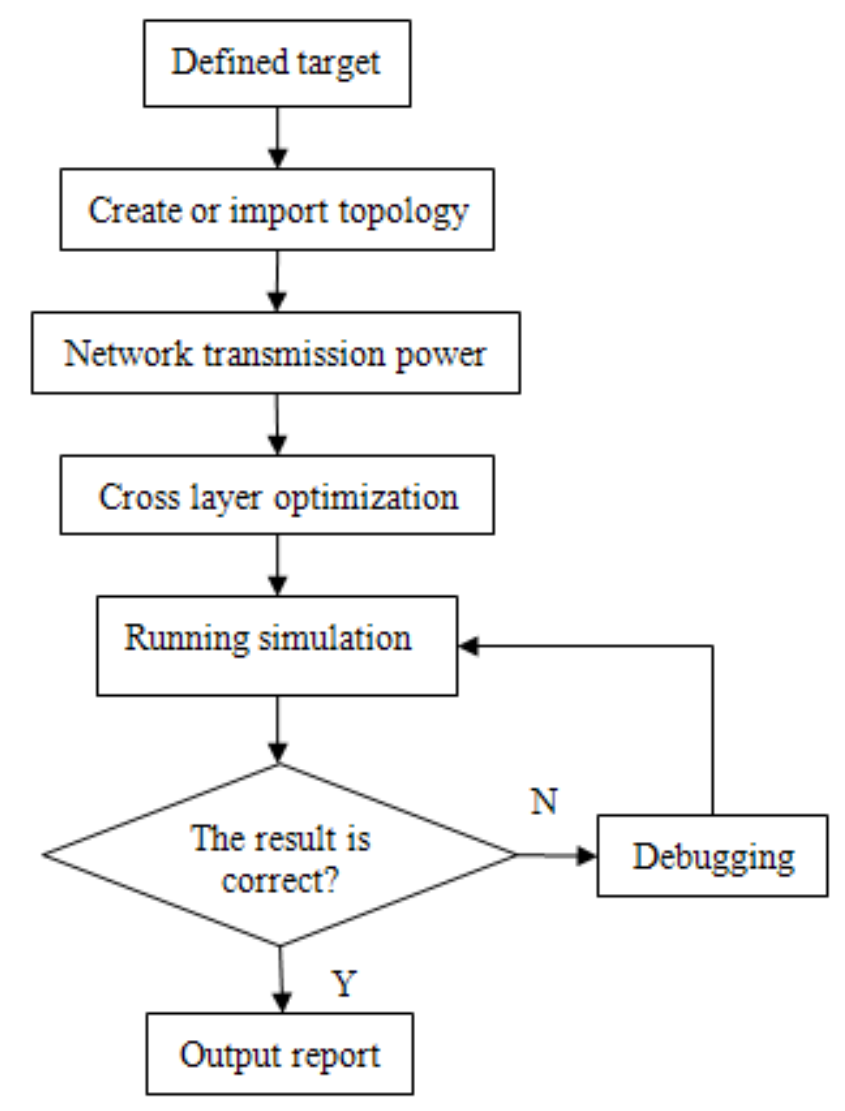

Fig.1 Flow chart of Wireless Ad Hoc Networks capacity and transmission power optimization

Figure 1 shows the capacity of wireless ad hoc networks and the transmission power of cross layer optimization. The process is divided into six steps. The first is to define the network, such as throughput capacity, link utilization, equipment efficiency, network delay, packet loss and other data, and then use OPNET software to establish a network simulation model, which includes self organization network, network topology and protocol. After the simulation model is built, the data is collected, and the simulation results are finished. Then, the network is optimized to carry out the cross layer optimization of base band capacity and transmission power, and then debug and simulation.

\section{Cross layer optimization of Baseband Design and transmission power for Wireless Ad Hoc Networks}

The baseband subsystem is also a part of the wireless self-organizing network. The baseband subsystem can receive data from multiple access subsystem, and the received data is sent to the digital intermediate frequency system. At the same time, the baseband subsystem receives the data which is processed by the intermediate frequency sub system, and the data is transmitted to the multiple access subsystem. In the actual communication process, from the signal transmitter to receive signal the end of the path and not only, thus cause multipath effect, the signal delay, increase transmission line load negatively, so as to reduce the transmission power, which influence the capacity of wireless ad hoc networks. In order to solve this problem, the OFDM technology is introduced into the network organization, within one OFDM symbol contains multiple sub signals via the modulation of the carrier wave, the symbol can be expressed as

$$
\begin{aligned}
& s(t)=\sum_{i=1}^{N-1} d_{i} \operatorname{rect}\left(t-t_{s}-T / 2\right) \exp \left(j 2 \pi f_{i}\left(t-t_{s}\right)\right), t_{s} \leq t \leq t_{s}+T \\
& \operatorname{rect}(t)=\left\{\begin{array}{c}
1,|t| \leq T / 2 \\
0, t=\text { others }
\end{array}\right.
\end{aligned}
$$


In this formula (1), $N$ is the number of subcarriers and $T$ is the symbol duration, $d_{i}$ represents the $i$-th sub signal load wave values, $f_{i}$ is the $i$-th sub carrier wave frequency, the formula of $f_{i}$ is as follows

$$
f_{i}=f_{c}+i / T
$$

The demodulation process is proformed for $m$ sub signals in the formula (1), in the t time, after the integral processing, the formula (3) can be obtained,

$$
\begin{aligned}
r & =\frac{1}{T} \int_{s}^{T+t_{s}} \exp \left[-j 2 \pi \frac{m}{T}\left(t-t_{s}\right)\right] \sum_{i=1}^{N-1} d_{i} \exp \left[-j 2 \pi \frac{i}{T}\left(t-t_{s}\right)\right] d t \\
& =\frac{1}{T} \sum_{i=1}^{N-1} d_{i} \int_{s}^{T+t_{s}} \exp \left[-j 2 \pi \frac{i-m}{T}\left(t-t_{s}\right)\right] d t=d m
\end{aligned}
$$

For the physical layer power control, the survival time and capacity of a wireless node depend on the battery capacity and power consumption, and the battery capacity can not be improved. The control of power and node capacity includes the introduction of the switch and the energy saving mode of the wireless network card, which can be realized by changing the clock frequency of CPU and the speed of the hard disk. The cross layer power control is the control of the transmission power to each protocol layer, and the optimized design of the network is realized by cross layer optimization. Cross layer optimization method is divided into two kinds, one is the low level of information feedback to high-level, such as the MAC layer of network data packet feedback to the transmission layer, so that the TCP protocol can distinguish the network congestion, the second method is to bundle many protocols, a protocol to another protocol.

\section{Simulation of wireless ad hoc network optimization design}

In order to improve the capacity and performance of wireless ad hoc networks, the architecture of computer and communication network is required to test the performance of the system. In order to implement the OPNET software, the simulation model of the wireless ad hoc network is built, which is shown in Figure 2.

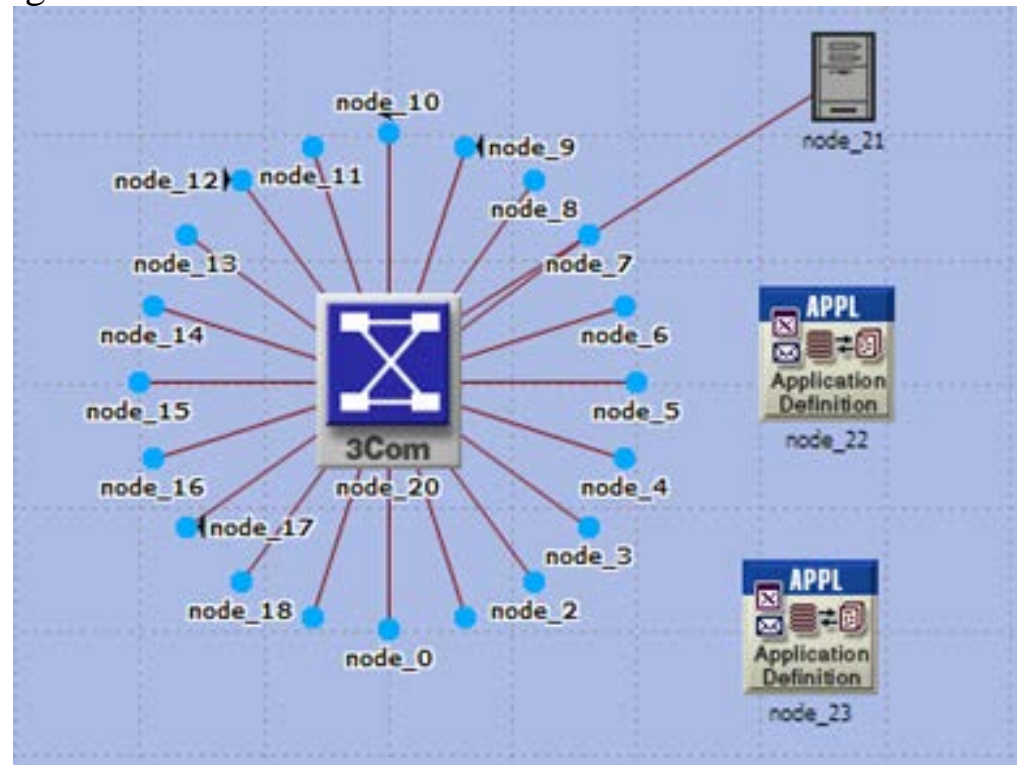

Fig. 2 OPNET wireless ad hoc network simulation model

Figure 2 shows that the wireless ad hoc network simulation model, which is built by OPNET software, is often a data packet loss problem in ad hoc networks. Using transmission power cross layer optimization method can effectively optimize the network, reduce the time to wait for a long time, improve the average throughput efficiency, to achieve the same effect as the packet loss. 


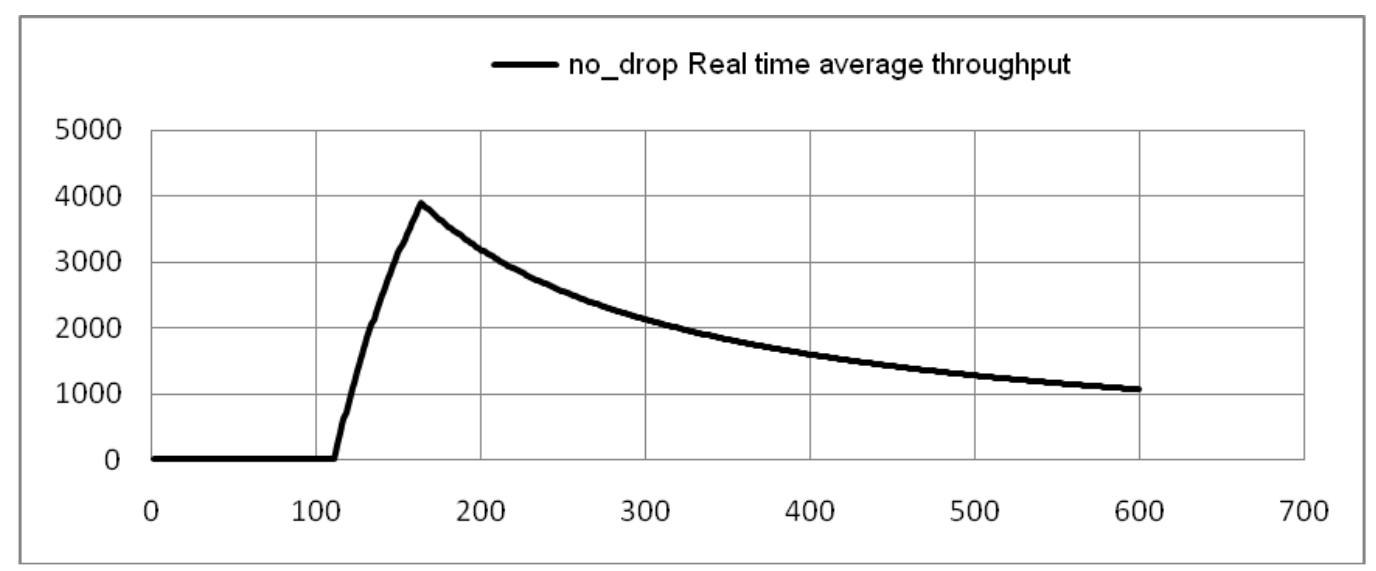

Fig. 3 The real-time average throughput rate of self organization network optimization

It shows in Fig. 3 that the average throughput curve of the real-time network under the optimized scene of the organization network. The first kind of method is used, the low level information feedback to the high level, the MAC layer of the network data packet feedback to the transmission layer, so that the TCP protocol can distinguish the network congestion, improve the network's capacity.

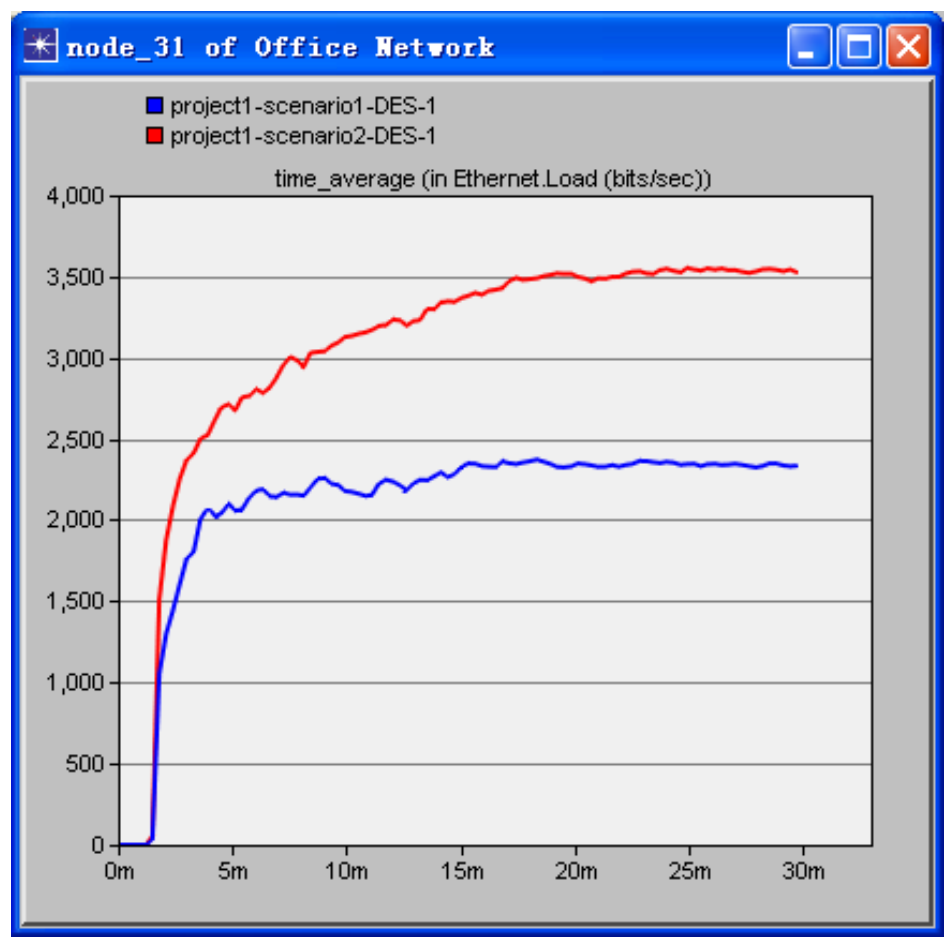

Fig. 4 The average load diagram before and after optimization

Figure 4 shows the average load curve of the network, which can be seen by the graph, the average load of the network is improved, and the load capacity of the network is improved.

\section{Conclusion}

Load capacity and power control is a typical cross layer optimization problem. A good load capacity and power control scheme can effectively reduce the power consumption of ad hoc networks, improve the capacity of network throughput, and effectively increase the transmission rate of packets. This paper presents a new method of cross layer transmission power optimization based on the base band design, which can be optimized according to the network system's constraints and network transmission characteristics. Using OPNET software for modeling and Simulation of the network, the network optimization method proposed in this paper can effectively control the network delay and achieve the same throughput capacity as the packet loss. 


\section{References}

[1] Lu Yanling,Wu Weiling. Application of smart antenna in CDMA network planning and optimization . Wireless communication technology, Vol.12 (1) , (2003): p.38 - 36

[2] Dai Qinyun, Hu Xiulin, Zhang Yunyu. Research on the capacity and delay performance of wireless AdHoc networks [J].Communication technology, Vol.31,(2),(2005): p.25-27.

[3] Yang Weijing, Zhang Jingzhou, Zhang Anxiang. Research and application of fuzzy adaptive PID control ,Computer Simulation, Vol.26 (9),(2009): p.132-135

[4] Xu Jun Jie, Chen Lei, Dong Xinmin. Based on ZigBee vibration monitoring wireless sensor network. Machine and hydraulic, Vol.38 (22) , (2010): p.108-109.

[5] Pang Na, Cheng Defu. Design of greenhouse monitoring system Based on ZigBee wireless sensor network. Journal of Jilin University, Vol.28 (1),(2010) : p.55-60

[6] Liu Jun, Li Zhe, Yue Lei. Design and implementation of self organization network simulation platform. computer science, Vol.35 (1),(2008): p.24-26,30.

[7] Zhao Xiaogang, Zhu Fengxian, Wu Di. Research and Realization of UAV Ad Hoc Network Simulation in a Rencounter Scenario. Journal of System simulation,Vol.20(23),(2008): p.6409-6413, 6428.

[8] Zhu Zhijun, Le Zichun, Zhu Ran. Research and implementation of OBS network simulation platform based on NS2 . Journal of communication, Vol.30 (9),(2009), p.128-134.

[9] Zhou Yiting, Ling Zhihao, Wu Qinqin.Discussion on ZigBee wireless communication technology and its application. Automation instrumentation, Vol.26 (6), (2005): p.5-9

[10] Dai Yuan, Yang Long, He Dongjian. Application of ZigBee in crop cultivation environment monitoring system. Micro computer information, Vol.25 (1-2), (2009): p.23-24

[11] Cao Yuanjun,Wang Xinzhong, Yang Jianquan. Research on. based on field meteorological monitoring system based on wireless sensor network, Vol.12,(2008): p.163-165

[12] Hui Nanmu, Li Yonggang, Wang Cheng. Wireless field measurement and control system based on Zigbee. Micro computer information, Vol.24 (8-3), (2008): p.52-53 